\section{In vitro differentiation of human amniotic epithelial cells into insulin- producing 3D spheroids}

\author{
Bernard Okere, ${ }^{1 *}$ Francesco Alviano, ${ }^{2 *}$ Roberta Costa, ${ }^{2}$ \\ Daniela Quaglino, ${ }^{3}$ Francesca Ricci, ${ }^{4}$ Massimo Dominici, ${ }^{5}$ \\ Paolo Paolucci,' Laura Bonsi' and Lorenzo lughetti'
}

International Journal of Immunopathology and Pharmacology 2015, Vol. 28(3) 390-402 (C) The Author(s) 2015 Reprints and permissions: sagepub.co.uk/journalsPermissions.nav DOI: $10.1177 / 0394632015588439$ iji.sagepub.com

@SAGE

\begin{abstract}
Regenerative medicine and stem cell therapy may represent the solution for the treatment of non-curable human diseases such as type I diabetes. In this context of growing demand for functional and safe stem cells, human amniotic epithelial cells (hAECs) from term placenta have attracted increasing interest for their wide availability, stem cell properties, and differentiation plasticity, which make them a promising tool for stem cell-based therapeutic applications. We initially assayed the stemness characteristics of hAECs in serum-free conditions. Subsequently we developed a culture procedure on extracellular matrix for the formation of three-dimensional (3D) spheroids. Finally, we tested the immunomodulation and differentiation potential of hAEC spheroids: the presence of pancreatic endocrine hormones was revealed with transmission electron microscopy and immunofluorescence analyses; the release of C-peptide in hyperglycemic conditions was assayed with ELISA.

The serum-free culture conditions we applied proved to maintain the basic stemness characteristics of hAECs. We also demonstrated that 3D spheroids formed by hAECs in extracellular matrix can be induced to differentiate into insulin-producing cells. Finally, we proved that control and induced cells equally inhibit the proliferation of activated mononuclear cells.

The results of this study highlight the properties of amnion derived epithelial cells as promising and abundant source for cell-based therapies. In particular we are the first group to show the in vitro pancreatic induction of hAECs cultured on extracellular matrix in a 3D fashion. We accordingly propose the outcomes of this study as a novel contribution to the development of future cell replacement therapies involving placenta-derived cells.
\end{abstract}

\title{
Keywords
}

3D culture, human amniotic epithelial cells, immunomodulation, insulin-producing cells, spheroids

Date received: 27 March 2015; accepted: 2 April 2015

\footnotetext{
'Division of Pediatric Oncology, Hematology and Marrow

Transplantation, Department of Medical and Surgical Sciences for Children \& Adults, University of Modena and Reggio Emilia, Modena Policlinic, Modena, Italy

${ }^{2}$ Department of Experimental, Diagnostic and Specialty Medicine, University of Bologna, Bologna, Italy

${ }^{3}$ Department of Life Sciences, University of Modena and Reggio Emilia, Modena, Italy

${ }^{4}$ Immunohematology and Transfusion Medicine Service, S. Orsola-

Malpighi Hospital, University of Bologna, Bologna, Italy
}

${ }^{5}$ Division of Oncology, Department of Medical and Surgical Sciences for Children \& Adults, University of Modena and Reggio Emilia, Modena Policlinic, Modena, Italy

*Equal contributors.

Corresponding author:

Bernard Okere, Division of Pediatric Oncology, Hematology and Marrow Transplantation, Department of Medical and Surgical Sciences for Children \& Adults, University of Modena and Reggio Emilia, Modena Policlinic, v. del Pozzo n.7I, Modena 4I 100, Italy.

Email: okere.b@hotmail.com 


\section{Introduction}

Regenerative medicine and stem cell therapy represent promising tools for the treatment of noncurable human diseases such as type 1 diabetes (T1D), which is characterized by the autoimmune destruction of pancreatic beta cells, resulting in the lack of insulin. The impairment of glucose homeostasis functions causes hyperglycemia and serious long-term complications. The shortage of transplantable pancreatic islets and the side effects of prolonged insulin injection to treat $\mathrm{T} 1 \mathrm{D},{ }^{1}$ have stimulated research into expandable and renewable sources of insulin-producing cells (IPCs). Stem cells hold great promise for supplying an adequate amount of donor cells for replacement therapies. In this connection, human embryonic stem cells (hESCs) and induced pluripotent stem (iPS) cells represent a virtually unlimited source of transplantable cells. Many studies have reported the derivation of IPCs from hESCs ${ }^{2}$ and iPS cells, ${ }^{3}$ but their future clinical application is hampered by the handling complexity, high cost of procedures, safety issues, and ethical concerns. ${ }^{4}$ Stem cells isolated from other tissues may surmount the issues related to the use of hESCs and iPS cells. Among them, placenta-derived stem cells are a promising source for cell transplantation and regenerative medicine. Human term placenta and fetal membranes are discarded after birth, so they can be easily obtained in large amounts without ethical concerns or invasive isolation procedures. Human amniotic epithelial cells (hAECs) are derived from human amniotic membrane and originate from epiblast at days 8-9 during embryological development, before gastrulation. ${ }^{5}$ hAECs express markers associated with hESCs and pluripotent stem cells, ${ }^{6}$ but unlike hESCs, hAECs are considered a safe alternative for cell therapies since they do not cause teratomas upon transplantation nor activation of the immune system. ${ }^{7}$ Moreover, hAECs have been successfully induced to differentiate towards mesodermic lineage, ${ }^{8}$ neural-ectodermic lineage, ${ }^{9}$ hepaticendodermic lineage, ${ }^{10}$ and pancreatic-endodermic lineage. ${ }^{11}$ For all these reasons hAECs are considered to retain most of the stem cell peculiarities of hESCs. In addition, the immune privilege of placental tissue grants hAECs the unique characteristic of being associated with poor antigenicity and immunomodulatory properties: in particular they inhibit mixed lymphocyte reactions as well as mitogen stimulated $\mathrm{T}$ cell proliferation in vitro ${ }^{12,13}$ and they do not induce immune reaction when transplanted in vivo. ${ }^{7}$

Previous studies successfully described the isolation and differentiation of hAECs into IPCs using classic or stepwise differentiation protocols, ${ }^{11}$ in the presence of media enriched with components of animal origin. The use of animal sera as growth supplement in human cell cultures might compromise the reproducibility and reliability of downstream assays and, therefore, could hamper the chance of clinical translation. ${ }^{14,15}$

Along with the requirements related to culture media composition, it is likewise worthy of consideration the adoption of culture methods that mimic the in vivo environment, such as three-dimensional (3D) culture systems. Several studies showed that stem cells cultured on 3D matrices enhance their ability to form higher-order structures like embryoid bodies, spheroids, and glandular-like acini. ${ }^{16,17}$ Moreover, 3D matrices enhance the stem cell potential and viability, while providing the suitable microenvironment for cell attachment, proliferation, and differentiation. ${ }^{18}$ These effects are a direct consequence of the synergic cell-to-cell and cellto-ECM interactions that cannot be provided in classical monolayer cultures. ${ }^{19,20}$ In particular, recent cell therapies studies for diabetes showed that $3 \mathrm{D}$ cultures could play a crucial role in the induction of hESCs into mature IPCs. ${ }^{21}$

In this perspective, in the present study we validated the stemness and immunomodulatory properties of hAECs in defined serum free conditions. We successfully developed a serum-free 3D culture procedure, based on extracellular matrix (ECM) hydrogel, for multicellular hAEC spheroid formation. Finally, for the first time in the field of placental stem cell research, we demonstrated that hAECs cultured in 3D serum-free conditions, functionally produce newly synthesized insulin in response to glucose stimulation.

\section{Materials and methods}

\section{Ethics}

The study was approved by the Local Research Ethics Committee (Azienda OspedalieroUniversitaria di Modena, prot. n 1171; 12/04/2010) and written informed consent was obtained from each healthy donor before specimen collection. 
Isolation and expansion of human amniotic epithelial cells (hAECs) in basal growth media (BM)

Amnion membranes were retrieved from term pregnancies (37-40 weeks of gestation) delivered by Cesarean section. hAECs were isolated using a modified procedure of the protocol previously reported by Ilancheran et al.:22 the amnion layer was mechanically peeled off the chorion layer and immediately washed in Hank's Balanced Wash Solution without calcium and magnesium (HBSS, PAA Laboratories $\mathrm{GmbH}$, Pasching, Austria) until blood clots were completely removed. The amnion was minced into small pieces $\left(4 \mathrm{~cm}^{2}\right.$ approximately) and incubated for $10 \mathrm{~min}$ at $37^{\circ} \mathrm{C}$ with $1 \mathrm{X}$ trypsin-EDTA (PAA Laboratories) to exclude debris. Membranes were then incubated for a second and third $40 \mathrm{~min}$ enzymatic digestion to release the amniotic epithelial cells. Single cell suspension was washed with Phosphate Buffered Saline (PBS, PAA Laboratories) and tested for viability with trypan blue dye (Biochrom AG, Berlin, Germany). Only samples with $>90 \%$ viability were used for further assays.

Cell pellets were resuspended in serum-free medium (SFM), which consisted of Quantum 286 (PAA Laboratories) serum-free medium for epithelial cells with $1 \%$ Penicillin-Streptomycin solution (PAA Laboratories) and epidermal growth factor (EGF) $(10 \mathrm{ng} / \mathrm{mL}$; Sigma-Aldrich, St. Louis, MO, USA), or serum-rich medium (SRM) made of DMEM (Invitrogen, Carlsbad, CA, USA) supplemented with heat-inactivated $10 \%$ fetal bovine serum (FBS) (PAA Laboratories), 1\% PenicillinStreptomycin solution (PAA Laboratories), and EGF (10 ng/mL; Sigma-Aldrich). The plating concentration in cell culture flasks was 80,000 cells/ $\mathrm{cm}^{2}$. Before further assays, the cells were allowed to attach to the substrate and grow in culture medium until 80-90\% confluency.

\section{Cell proliferation assay}

In order to evaluate cell proliferation potential, hAECs were plated at 60,000 cells $/ \mathrm{cm}^{2}$ in 24-well plates and cell growth was assessed using Alamar Blue assay. Alamar Blue 10\% (Invitrogen) was added to basal medium (BM) for $4 \mathrm{~h}$ and then fluorescence was measured using Victor Multilabel Counter (Perkin Elmer, Boston, MA, USA). The same procedure was repeated on days $3,5,8,10$,
12, and 15. Data were plotted as Fluorescence Arbitrary Unit (AU fluorescence) analyzed at specific time points.

\section{Flow cytometric characterization of hAECs}

Flow cytometric analysis was performed on primary cultures of hAECs. Cells were detached with Trypsin-EDTA and incubated with antibodies $\left(1 \mu \mathrm{g} / \mathrm{mL}\right.$ for $30 \mathrm{~min}$ at $\left.4^{\circ} \mathrm{C}\right)$. The antibodies used were: anti-CD105-FITC (Beckman Coulter, Miami, FL, USA), anti-panCytokeratin-PE (Santa Cruz Biotechnology, Santa Cruz, CA, USA), antiSSEA-4-APC (R\&D Systems, Minneapolis, MN, USA), anti-OCT4 primary antibody (Santa Cruz Biotechnology), and secondary anti-mouse IgGFITC (Beckman Coulter). Cells were then washed and analyzed on a flow cytometer (Navios FC, Beckman Coulter); acquired data were elaborated with Kaluza FC Analysis software.

\section{RNA isolation, reverse transcription and real-time $P C R$ ( $q P C R)$}

Total RNA was extracted from hAECs grown in culture flasks using the PureLink RNA Mini Kit (Invitrogen) according to the manufacturer's instructions. cDNA was synthesized from 1-2 $\mu \mathrm{g}$ of total RNA using RETROscript First Strand Synthesis Kit and oligo dT (Invitrogen). Oligonucleotide pairs were designed using qPCR SciTools software (Integrated DNA Technologies). Primer sequences and gene accession numbers for stemness gene markers (Ki67, OCT4a, NANOG, SOX2) are listed in Table 1. qPCR assays were performed in a StepOne System (Applied Biosystems, Foster City, CA, USA) with KAPA SYBR FAST Master Mix (KAPA BIOSYSTEMS, Woburn, MA, USA). Data were normalized against the internal control beta$\operatorname{actin}(\mathrm{ACTB})$.

\section{Immunomodulatory properties of hAECs}

In order to evaluate the immunomodulatory potential of hAECs on activated peripheral blood mononuclear cells (PBMCs), hAECs were plated in 6 -well plates $\left(60,000\right.$ cells $\left./ \mathrm{cm}^{2}\right)$ in SFM or SRM, and were allowed to stabilize in culture for 7 days. Then, PBMCs were isolated from healthy donors by density gradient centrifugation (Ficoll-Paque 1077, Sigma-Aldrich) and plated on hAEC monolayer at a 
Table I. PCR primers and gene accession number.

\begin{tabular}{llll}
\hline Gene & Forward primer & Reverse primer & Accession no. \\
\hline Ki67 & TCC TAA TAC GCC TCT CAA AAG & GAT TTC TGA ACC TGA CTC TTG & NM_00II45966 \\
OCT4a & AGA GAA AGC GAA CCA GTA TC & TTA CAG AAC CAC ACT CGG & NM_00270I \\
NANOG & AGA AAT ACC TCA GCC TCC AG & CGT CAC ACC ATT GCT ATT CTT & NM_024865 \\
SOX2 & CAG ACT TCA CAT GTC CCA G & CTC CCA TTT CCC TCG TTT & NM_003I06 \\
BETA-ACTIN & CCT TCT ACA ATG AGC TGC G & CCT GGA TAG CAA CGT ACA TG & NM_00II0I \\
\hline
\end{tabular}

density of $2 \times 10^{6}$ cells/well in RPMI 1640 (Lonza, Walkersville, MD, USA). PBMCs were activated by addition of Phytohemagglutinin (PHA, $5 \mu \mathrm{g} / \mathrm{mL}$, Sigma-Aldrich) and incubated for $72 \mathrm{~h}$ at $37^{\circ} \mathrm{C}, 5 \%$ $\mathrm{CO}_{2}$. Finally PBMCs were resuspended, recovered, and fixed with $70 \%$ ethanol at $4{ }^{\circ} \mathrm{C}$, stained with Propidium Iodide (Beckman Coulter) at room temperature for $10 \mathrm{~min}$. The negative control consisted of PBMCs without PHA stimulation, while positive control was PBMCs with PHA $(5 \mu \mathrm{g} / \mathrm{mL})$ in the absence of hAECs. Cell cycle phase distribution of PBMCs was analyzed by flow cytometer.

\section{Three-dimensional (3D) culture}

3D culture of hAECs was performed according to the overlay method previously described by Debnath et al. ${ }^{16}$ Briefly, isolated hAECs were first resuspended in SFM added with $2.5 \%$ reduced growth factor basement membrane extract (BME) from Engelbreth-Holm-Swarm (EHS) mouse sarcoma (Trevigen, Gaithersburg, MD, USA). Then, resuspended cells were seeded $\left(60,000 \mathrm{cells} / \mathrm{cm}^{2}\right)$ on a thick layer of $100 \%$ reduced growth factor BME measuring approximately $1 \mathrm{~mm}$ in thickness. Multicellular spheroids formed after 5-6 days, partially embedded in the gelled layer of basement membrane. Cell culture medium was changed every 3 days. hAEC spheroids were harvested and collected for further assays using Cultrex 3D culture cell harvesting kit (Trevigen Inc.).

\section{In vitro pancreatic stepwise differentiation}

Prior to differentiation, hAECs were allowed to grow for 5 days in SFM until the sufficient spheroid size. Pancreatic induction of 3D cultured cells was performed using a stepwise protocol divided into three stages: Stage 1: hAEC spheroids were predifferentiated for 4 days in SFM supplemented with $100 \mathrm{ng} / \mathrm{mL}$ Activin A; Stage 2: SFM was supplemented with $100 \mathrm{ng} / \mathrm{mL}$ Activin A and Nicotinamide
(NAM, 10mM) for 4 additional days; and Stage 3: terminal differentiation was performed switching the medium to SFM with NAM $(10 \mathrm{mM})$ and Retinoic Acid (RA) $(2 \mu \mathrm{M})$ for 10 days. Glucose concentration of SFM was $\geqslant 15 \mathrm{mM}$. This hyperglycemic culture condition is suitable for epithelial cells growth and was maintained for pancreatic differentiation assay to enhance the induction of hAECs into IPCs. ${ }^{23}$ Media supplements were all obtained from Sigma-Aldrich.

\section{Immunofluorescence analysis and confocal microscopy}

3D hAEC spheroids were fixed with $2 \%$ Paraformaldehyde (PFA, Sigma-Aldrich) for 20 min at room temperature. After washing $2 \times 10 \mathrm{~min}$ in PBS, spheroids were permeabilized in $0.5 \%$ Triton X-100 (Sigma-Aldrich) in PBS for $10 \mathrm{~min}$ and then rinsed again $2 \times 10 \mathrm{~min}$ in PBS. Antigen blocking was performed for $1 \mathrm{~h}$ at room temperature in blocking solution: 5\% normal goat serum (Vector Laboratories, Burlingame, CA, USA), 0.2\% Triton $\mathrm{x}-100,0.1 \%$ Bovine Serum Albumin (BSA) (Sigma-Aldrich). Primary and secondary antibodies were diluted in freshly prepared blocking solution. Primary antibodies were incubated at $8^{\circ} \mathrm{C}$ overnight and secondary antibodies for $40-50 \mathrm{~min}$ at room temperature. The following primary antibodies and dilutions were used: mouse anti-insulin 1:100 (Abcam ab46707); rabbit anti-glucagon (Abcam ab8055); rabbit anti-C-peptide 1:100 (Cell Signalling \#4593); rabbit anti-CK19 1:100 (Thermo Scientific PA5-16726); mouse antilaminin 1:100 (Thermo Scientific MA1-21194). Secondary antibodies were: DyLight 594 goat antimouse 1:200 (Jackson Immuno Research Labs.); Alexa Fluor 488 goat anti-rabbit 1:400 (Molecular Probes); CF 488 goat anti-mouse 1:400 (Biotium); DyLight 594 goat anti-rabbit 1:200 (Thermo Scientific). Nuclei counterstaining was performed with Fluoroshield+DAPI (Sigma-Aldrich). Imaging 
was performed by confocal microscopy (Leica TCS SP2, Leica Microsystems, Mannheim, Germany) and images were processed using Leica Confocal Software.

\section{Transmission electron microscopy (TEM)}

Pancreatic induced hAECs cultured in 3D matrices and undifferentiated control cells were gently washed with Tyrode's buffer, $\mathrm{pH}$ 7.3, and centrifuged at $10,000 \mathrm{x}$ g for $5 \mathrm{~min}$ at $10^{\circ} \mathrm{C}$. The resulting pellets were fixed overnight with $2.5 \%$ Glutaraldehyde (Electron Microscopy Sciences, Hatfield, PA, USA) in Tyrode's buffer, postfixed for $2 \mathrm{~h}$ in $1 \%$ Osmium Tetroxide (Electron Microscopy Sciences), dehydrated, and embedded in Spurr resin (Electron Microscopy Sciences). Since cells were not homogeneously distributed within matrices, semi-thin sections were routinely stained with Toluidine Blue and observed with a Zeiss Axiophot light microscope (Carl Zeiss, Jena, Germany) to locate cells in each cut layer. Ultrathin sections were performed on areas selected for the presence of spheroids and observed with a Jeol 2010 electron microscope (Jeol, Tokyo, Japan) at $200 \mathrm{kV}$ after staining with Uranyl Acetate and Lead Citrate (Electron Microscopy Sciences).

\section{Glucose-stimulated release and expression of C-peptide}

To confirm the synthesis of insulin, C-peptide release from differentiated and undifferentiated hAEC spheroids was measured in hypoglycemic and hyperglycemic conditions. First, cells were incubated in Krebs-Ringer solution with bicarbonate and HEPES (KRBH; $129 \mathrm{mM} \mathrm{NaCl}, 4.8 \mathrm{mM}$ $\mathrm{KCl}, 2.5 \mathrm{mM} \mathrm{CaCl} 2,1.2 \mathrm{mM} \mathrm{K \textrm {K } _ { 2 }} \mathrm{PO}_{4}, 1.2 \mathrm{mM}$ $\mathrm{MgSO}_{4}, 5 \mathrm{mM} \mathrm{NaHCO}, 10 \mathrm{mM}$ HEPES, 0.1\% (wt/vol) BSA) (Sigma-Aldrich) for $70 \mathrm{~min}$ as a preliminary washing step. Next, spheroids were incubated in $\mathrm{KRBH}$ with $2 \mathrm{mM}$ D-Glucose (SigmaAldrich) for $1 \mathrm{~h}$ and then switched to hyperglycemic stimulation condition consisting of KRBH with $20 \mathrm{mM}$ D-Glucose and $30 \mathrm{mM} \mathrm{KCl}$ for $1 \mathrm{~h}$. C-peptide levels in supernatant from basal $(2 \mathrm{mM}$ D-Glucose) and stimulation condition (20 mMDGlucose $+30 \mathrm{mM} \mathrm{KCl}$ ) were measured using the Human Ultrasensitive C-peptide enzyme-linked immunosorbent assay (ELISA) kit (Mercodia, Uppsala, Sweden) and normalized to total amount of cellular DNA using CyQUANT Cell Proliferation Assays Kit (Invitrogen). The fold stimulation was calculated as the ratio of the C-peptide concentration in supernatant from hyperglycemic condition to the C-peptide concentration from hypoglycemic condition. Glucose-stimulated spheroids were immediately assayed for the presence of C-peptide with immunofluorescence analysis.

\section{Immunomodulatory activity of hAEC spheroids}

The immunomodulatory activity of hAECs was analyzed after stepwise pancreatic-endocrine differentiation in 3D culture. PBMCs were isolated as previously described and plated on hAEC spheroids at the end of pancreatic differentiation protocol (hAECs/PBMCs ratio 1:2). PBMCs were activated by addition of Phytohemagglutinin (PHA) $(5 \mu \mathrm{g} /$ $\mathrm{mL}$; Sigma-Aldrich) and hAEC spheroids/PBMCs co-cultures were incubated for $72 \mathrm{~h}$ at $37^{\circ} \mathrm{C}, 5 \%$ $\mathrm{CO}_{2}$. The negative control consisted of PBMCs without PHA stimulation and the positive control was PBMCs with PHA $(5 \mu \mathrm{g} / \mathrm{mL})$ in the absence of hAEC spheroids. Finally PBMCs were resuspended, recovered, and plated in 96-wells plate for BrdU incorporation analysis. BrdU incorporation was evaluated using a colorimetric immunoassay, according to the manufacturer's instructions (Cell Proliferation ELISA, BrdU - colorimetric kit, Roche, Basel, Switzerland).

\section{Statistical analysis}

The assays were repeated a minimum of three times independently and the results were expressed as the mean $\pm \mathrm{SD}$. Statistical analysis was performed using two-tailed, paired T-test, or one-way ANOVA test. $P<0.05$ was considered to be statistically significant.

\section{Results}

\section{hAEC epithelial morphology and proliferation} rate

hAECs were isolated from term placenta and grew as adherent cells when seeded in classical serumrich medium (SRM) or in serum-free medium (SFM). In both culture conditions hAECs regularly proliferated and formed a confluent monolayer of cells with epithelial cobblestone-shaped morphology (Figure 1a). In order to evaluate the culture 
medium influence on hAEC growth kinetics and proliferation ability, we performed a cell growth assay for 15 days. The resulting growth curve showed a similar proliferation rate for hAECs in both SRM and SFM (Figure 1b).

\section{Essential characterization of SFM-cultured hAECs}

To evaluate the effects of culture media, we tested whether serum deprivation could influence hAEC stemness features and immunomodulatory properties. First, we examined the expression of a selected antigenic surface pattern (Figure 2a). Flow cytometric analysis displayed a similar hAECs immunophenotype for both cell culture media: protein expression levels of stemness markers, OCT4 and SSEA-4, were clearly positive (OCT4: $99.1 \pm 0.2 \%$ in SRM and $99.3 \pm 0.3 \%$ in SFM; SSEA-4: $97.3 \pm$ $0.4 \%$ in SRM and $98.9 \pm 0.2 \%$ in SFM); characteristic mesenchymal antigen CD105 was expressed $(94.5 \pm 0.5 \%$ in SRM and $93.7 \pm 0.6 \%$ in SFM) as previously reported; ${ }^{8}$ distinctive epithelial marker PanCK was uniformly expressed in the hAECs population $(99.9 \pm 0.1 \%$ in SRM and $99.9 \pm 0.1 \%$ in SFM). These data proved that SFM culture does not affect the immunophenotype of hAECs.

Moreover, qPCR was carried out to test for serum-induced changes in the expression of proliferative gene Ki67 and stemness markers NANOG, SOX2, and OCT4a. Ki67 mRNA decrease in serum-free medium is not significant, while gene expression levels for stemness markers were clearly and significantly higher in serum-free conditions than in serum-rich media, namely 70 -fold for NANOG, 36-fold for SOX2, and 12-fold increase for OCT4a mRNA (Figure 2b).

Finally, to assess hAEC immunomodulatory ability in serum-free culture conditions, PHAstimulated PBMCs were co-cultured with hAECs in SRM and SFM, and their proliferation was assayed to test hAEC immunomodulatory properties. The distribution of PBMCs in the cell cycle phases was evaluated (Figure 2c). Non-activated PBMCs were all in G1-G0 phase $(96.4 \pm 0.3 \%)$, while activated PBMCs in the absence of hAECs significantly decreased in the G1-G0 phase (68.7 \pm $3.3 \%)$ and increased in the $\mathrm{S}(18.6 \pm 0.5 \%)$ and G2-M $(12.1 \pm 2.0 \%)$ phases $(P<0.05)$. After coculture with hAECs in SRM or SFM, we observed a significant increase of PBMCs in G1-G0 (83.0 \pm $4.1 \%$ in SRM and $82.7 \pm 4.9$ in SFM) and an equally

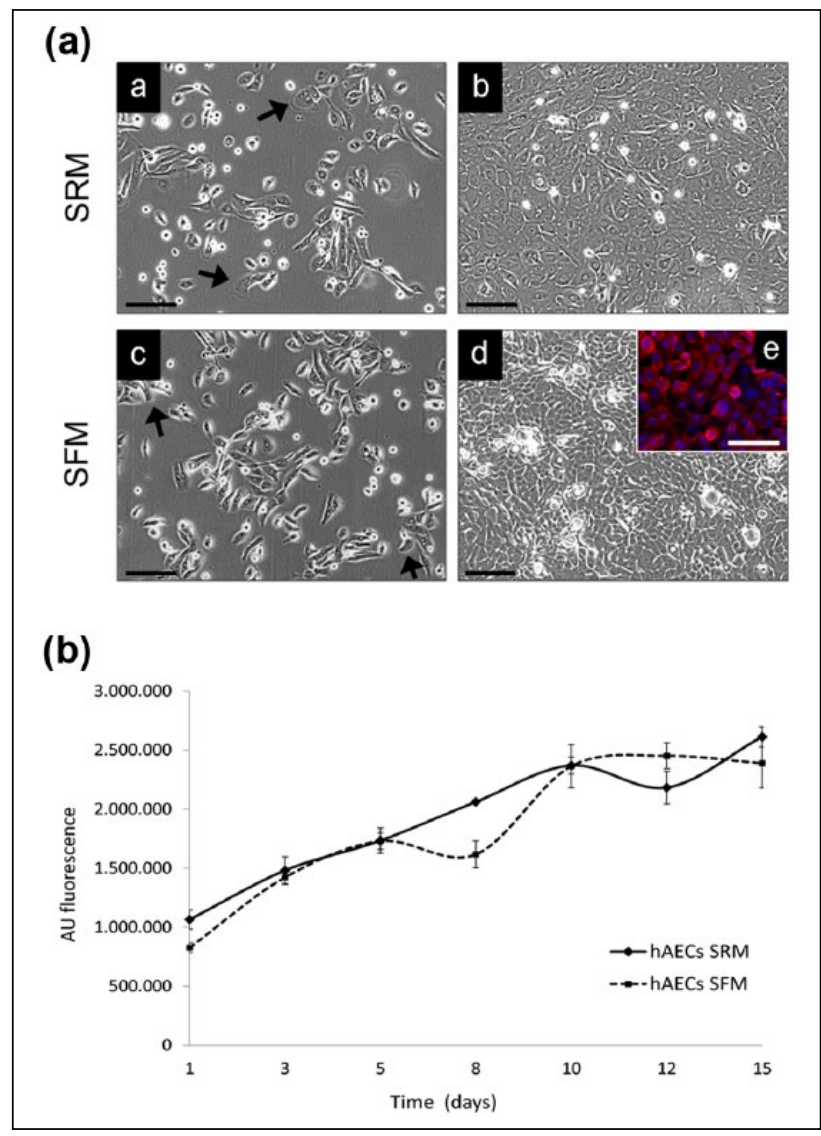

Figure I. hAECs retain distinctive epithelial morphology and regular growth in serum free medium. (a) Phase-contrast morphology of hAECs grown as a monolayer in serum rich (SRM) $(a, b)$ and serum free (SFM) culture media (c, d). Subconfluent hAECs (24 h following isolation) grew as isolated cells and clusters $(a, c)$. Lamellipodia are visible (black arrows). At confluence, hAECs assumed a cobblestone morphology, typical of epithelial cells (b, d). Representative immunofluorescence (e) image shows hAEC immunoreactivity for CK 19 marker; nuclei are counterstained with DAPI. Scale bars $=50 \mu \mathrm{m}$. (b) In vitro expansion of hAECs in SRM and SFM culture media. Cells were isolated and seeded at 60,000 cells $/ \mathrm{cm}^{2}$, and cell proliferation was analyzed for 15 days. Data are expressed as arbitrary units of fluorescence (AU Fluorescence). Means $\pm S D ; n=3$.

sizable reduction of PBMCs in the $\mathrm{S}(11.5 \pm 1.7$ in SRM and $12.0 \pm 0.6 \%$ in SFM) and G2-M (4.4 \pm $2.6 \%$ in SRM and $3.7 \pm 2.6 \%$ in SFM) phases $(P$ $<0.05)$. We may assume from these observations that hAECs cultured in either SRM or SFM, similarly reduce the proliferation of stimulated PBMCs.

\section{Morphogenesis of hAEC spheroids grown in 3D culture}

When cultured on a thick bed consisting of $100 \%$ gelled basement membrane extract (BME), hAECs adopted a spheroid, 3D morphology (Figure 3a). 
(a)
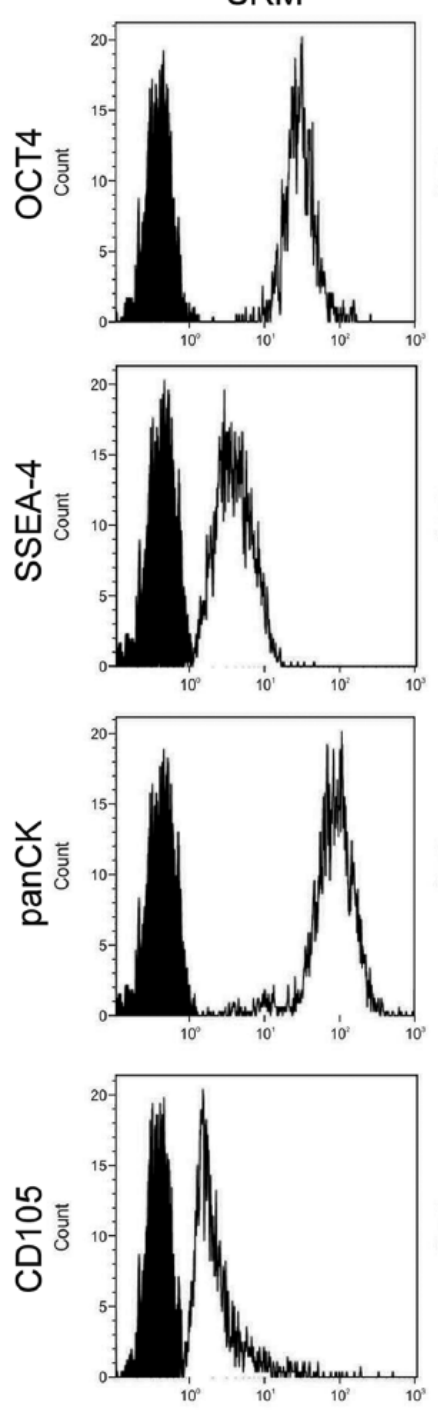
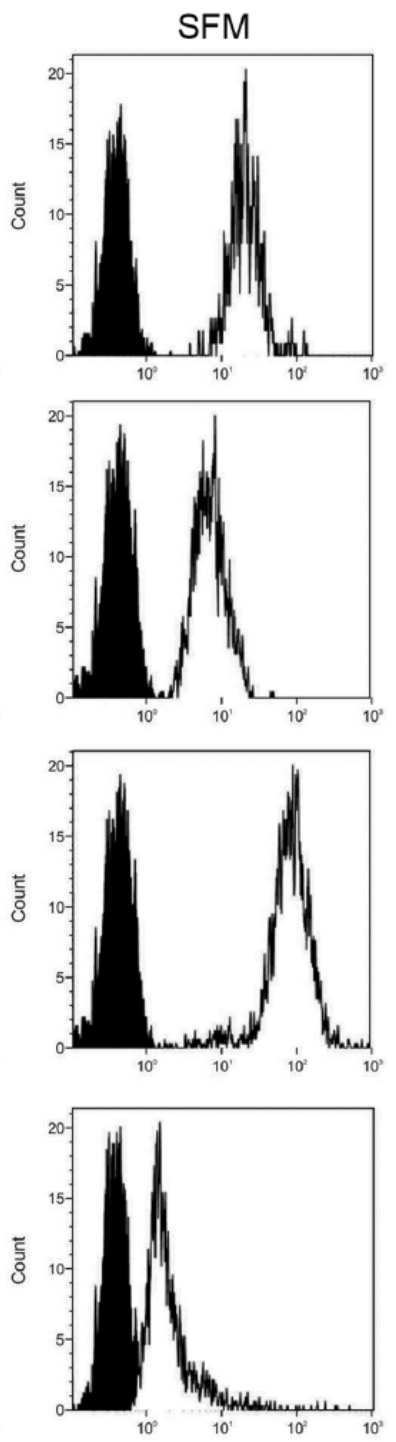

(b)

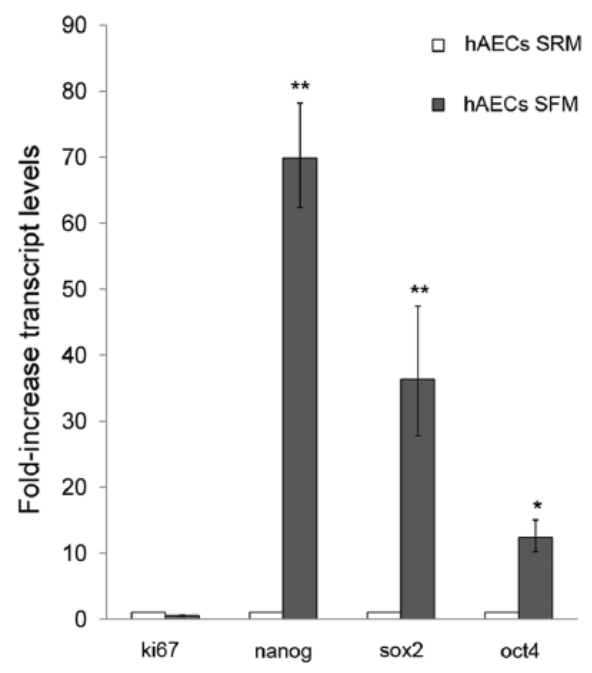

(c)

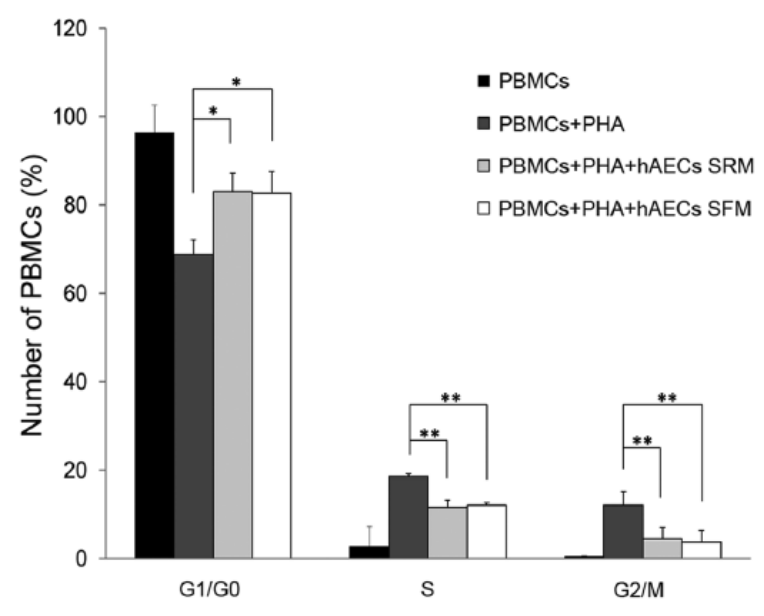

Figure 2. Stemness features and immunomodulatory properties of hAECs in SFM. (a) Immunophenotypical analysis of hAECs cultured in SRM and in SFM. Primary cultures of hAECs were stained with antibodies against Oct 4, SSEA-4, CDI05, and PanCK. Representative images were chosen from five independent immune phenotyping experiments. (b) Gene expression levels for Ki67, NANOG, SOX2, and OCT4a. mRNAs contents of hAECs cultured in SFM (black bars) are expressed as the fold increase against control cells cultured in SRM (white bars). Means \pm SD; $n=5$. *P<0.05; **P<0.01. (c) Analysis of PBMC distribution in cell cycle phases after co-culture with hAECs. Co-culture of PHA-activated PBMCs with hAECs in SRM or SFM reduced PBMC proliferation. Data are expressed as a percentage of PBMCs for each cell cycle phase. Means $\pm S D ; n=6$. $* P<0.05$; $* * P<0.0$ I

After 1 week of culture, we observed the formation of multicellular structures which increased in complexity, size, and cellular density over time, reaching a complete and definitive spheroid architecture $(>40 \mu \mathrm{m})$ around days $10-12$. The immunofluorescence analysis (Figure $3 \mathrm{~b}$ ) highlights the peculiar laminin localization at the cell mass outer perimeter, separating the spheroid from the surrounding extra cellular matrix. Laminin spatial arrangement, suggests the acquisition of a correct apico-basal polarization by hAEC spheroids. In addition, SFM was more effective in maintaining a stable spheroid morphology after 2 weeks of culture, while the serum-rich conditions caused the development of irregular and larger cell clusters (data not shown). 
(a)
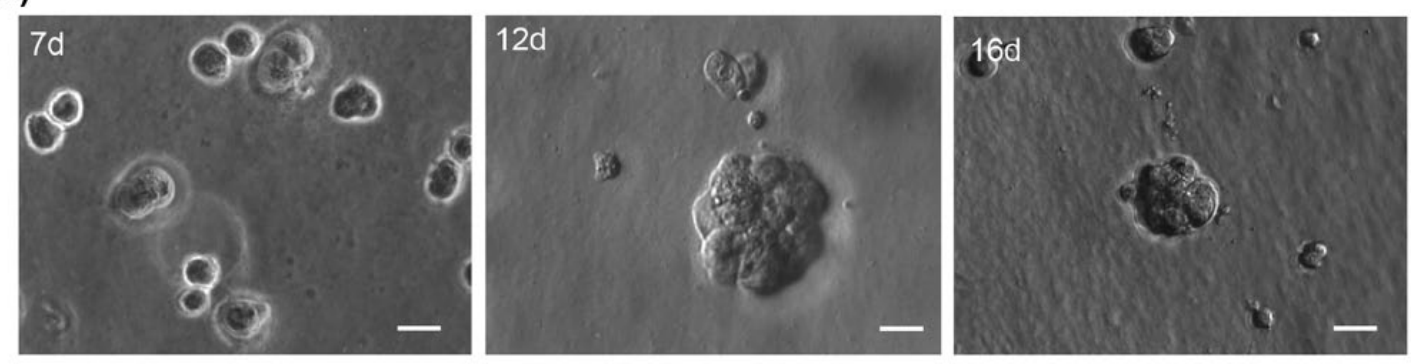

(b)

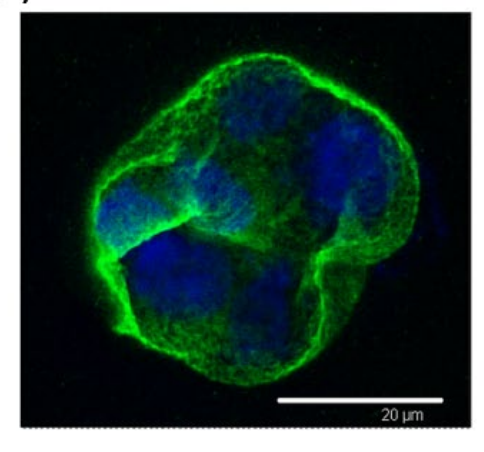

Figure 3. Development of hAEC spheroids. (a) Phase contrast micrograph of three-dimensional (3D) hAEC spheroids cultured on basement membrane at 7, 12, and 16 days in SFM. hAEC spheroids reached a definitive architecture after 10-12 days. (b) Confocal immunofluorescence analysis revealed the encircling distribution of laminin. Nuclei are counterstained with DAPI. Scale bars $=20 \mu \mathrm{m}$.

\section{hAEC spheroids inhibit proliferation of mitogen- activated PBMCs}

In order to investigate if hAEC immunomodulatory ability was maintained even after pancreatic differentiation in $3 \mathrm{D}$ culture system, we co-cultured hAEC differentiated spheroids with PHAstimulated PBMCs and measured BrdU incorporation levels in PBMCs (Figure 4). Non-activated PBMCs do not incorporate BrdU $(2.8 \pm 1.6 \%)$, while PHA-activated PBMCs regularly incorporate high levels of $\mathrm{BrdU}(98.6 \pm 1.4 \%)$. BrdU incorporation levels significantly decreased after co-culture with induced $(8.1 \pm 5.7 \%)$ or non-induced $(8.5 \pm$ 4.4\%) hAEC spheroids $(P<0.05)$. To conclude, no significant differences in inhibition activity on PBMC proliferation were observed between differentiated and non-differentiated hAEC spheroids.

\section{Differentiated hAEC spheroids express} pancreatic hormones and display islet-like ultrastructure

To determine whether our stepwise differentiation protocol efficiently induced hAEC spheroids into

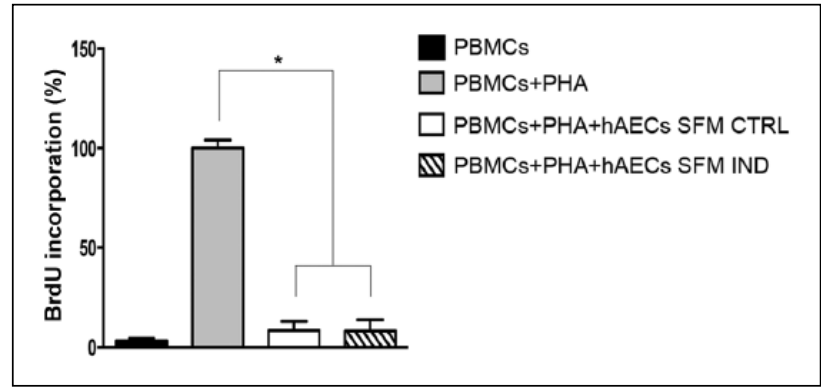

Figure 4. Immunomodulatory activity of hAEC spheroids. Co-culture of PHA-activated PBMCs with control and differentiated hAEC spheroids in SFM led to significant reduction in PBMC proliferation assayed by $\mathrm{BrdU}$ incorporation levels. Control and differentiated hAECs equally suppressed proliferation of PHA-stimulated PBMCs. Data are expressed as a percentage of $\mathrm{BrdU}$ incorporation. Means $\pm \mathrm{SD}$; $\mathrm{n}=6 . * \mathrm{p}<0.05$.

hormone-producing cells, we performed immunofluorescence analysis and checked for the presence of pancreatic hormones: glucagon and insulin (Figure 5a). Basal expression of glucagon was present in undifferentiated control cells (data not shown), but glucagon immunoreactivity became more intense upon differentiation. Basal expression 
(a)
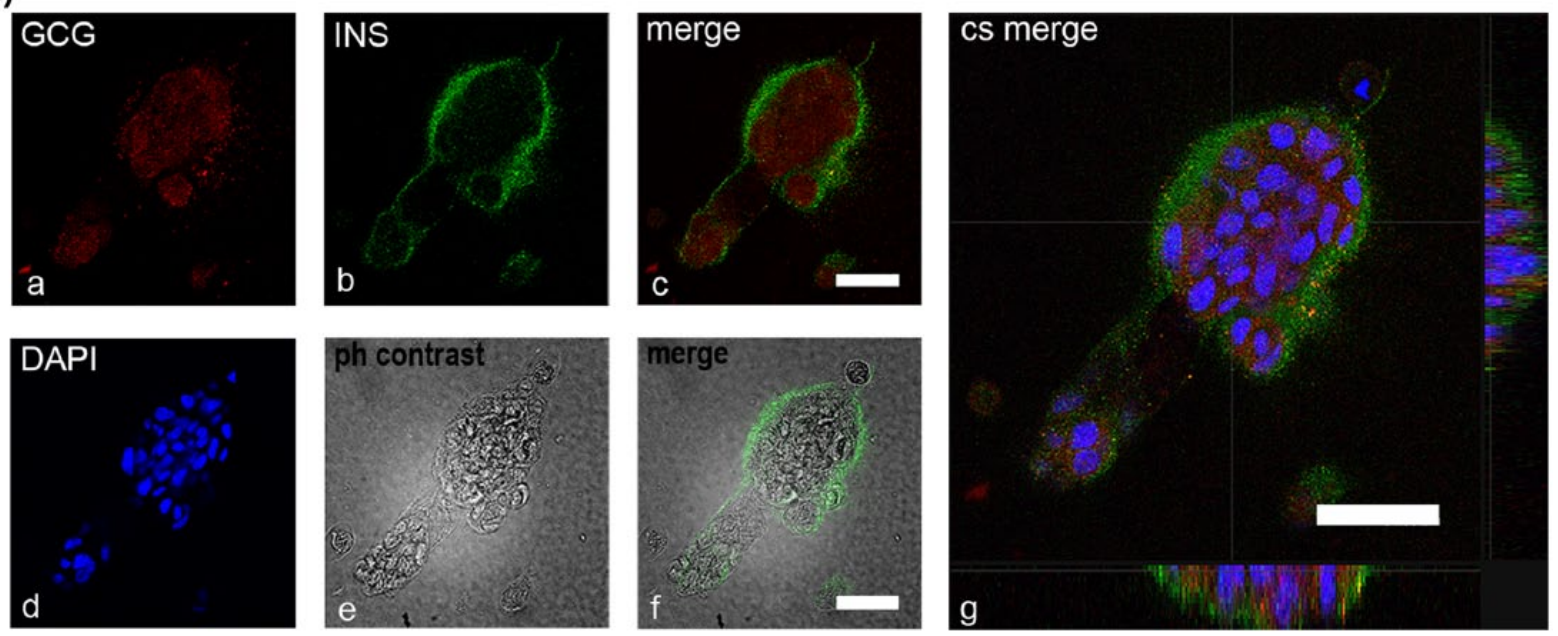

(b)

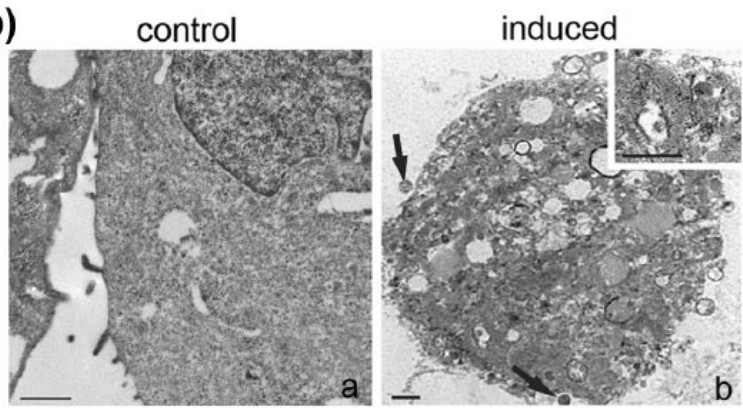

Figure 5. Pancreatic-endocrine induced hAECs express insulin. (a) Confocal immunofluorescence analysis of hormoneexpressing cells. Insulin and glucagon staining of hAEC spheroids in SFM (a-g). Insulin was localized in the peripheral areas of the spheroid while glucagon was located in the central mass. Phase-contrast micrograph (e) shows the structure of hAEC spheroid. Merge micrograph (f) highlights the strict perimembrane localization of insulin (b). The overlays (c, g) show the co-existence of insulin (b) and glucagon (a), expressed in different parts of the spheroid. In particular, cross-section merge micrograph (g) show plan $x$ and plan $y$ spatial organization of insulin and glucagon. Nuclei are counterstained with DAPI (d). Scale bars $=40 \mu \mathrm{m}$ (c, $\mathrm{f}$, g). (b) Electron micrograph of control hAECs showing normal cytoplasmatic ultrastructure (a) and pancreatic-endocrine induced hAEC spheroid cultured in SFM (b) showing granules with crystalline electron-dense cores, reminiscent of beta cell granules (black arrows). Scale bars $=\mathrm{I} \mu \mathrm{m}$.

of insulin was absent from undifferentiated hAECs. After 20 days of pancreatic induction in serum-free conditions, spheroids became insulin-positive. Confocal micrographs revealed the external localization of insulin-positive cells: merged images of immunofluorescence and phase-contrast micrographs showed the membrane-related position of insulin, while glucagon was exclusively expressed in hAECs localized in the central area of the spheroids. The peripheral arrangement of insulin is consistent with the exocytosis mechanism for the release of insulin granules in response to high glucose levels.

To assess the endogenous production of insulin and verify whether induced-hAECs formed mature secretory granules we used transmission electron microscopy (TEM) (Figure 5b). TEM ultrastructural analysis of induced hAECs (versus undifferentiated control cells) revealed the high complexity of the cytoplasm and the presence of several vesicles, consistent with intense protein synthesis. In particular, cells contained various secretory granules with a clear halo surrounding an electrondense core, which is peculiar to mature insulincontaining granules.

\section{C-peptide release and expression in hAEC spheroids}

To further confirm the endogenous nature of the detected insulin, C-peptide secretion assay was performed in differentiated and non-differentiated 
spheroids (control group) (Figure 6a). Basal secretion of C-peptide was measured in control group at $2 \mathrm{mM}$ D-glucose and $20 \mathrm{mM}$ D-glucose concentrations $(0.227 \pm 0.027 \mathrm{pmol} / \mathrm{ng}$ DNA and $0.244 \pm$ $0.033 \mathrm{pmol} / \mathrm{ng}$ DNA, respectively). Pancreaticendocrine differentiated spheroids secreted higher levels $(P<0.02)$ of C-peptide in $20 \mathrm{mM}$ D-Glucose condition $(0.697 \pm 0.036 \mathrm{pmol} / \mathrm{ng} \mathrm{DNA})$, than differentiated spheroids in $2 \mathrm{mM}$ D-glucose condition $(0.427 \pm 0.050 \mathrm{pmol} / \mathrm{ng}$ DNA). These data further support the success of the differentiation protocol and show the acquisition of a glucose-stimulated insulin release mechanism from hAEC spheroids. Subsequently, immunofluorescence analysis performed on differentiated hAEC spheroids, revealed a clear and widespread presence of C-peptidepositive cells (Figure 6b).

\section{Discussion}

In this study, we propose a novel set of procedures to induce placental-derived hAECs into insulinproducing cells (IPCs), combining different cell culture approaches in order to overcome issues such as the presence of animal serum in the media composition and cell differentiation inefficiency.

Effective and safe cell therapies require strictly standardized and GMP operating methodologies, including optimal clinically compliant culture procedures. ${ }^{14,15}$ Most of the current in vitro data obtained from studies on stem cells were generated under artificial conditions using standard culture media containing FBS, which may impair cell culture assay reproducibility, alter any experimental outcome, and delay the clinical translation.

In this regard, we first validated serum free culture condition from cell isolation to cell differentiation: we concisely confirmed the outcomes from previous studies $^{6,8}$ and extended the observations to our serum-free conditions. The unaltered epithelial morphology, the positive immunophenotype profile of key stemness markers (OCT4, SSEA-4), the growth kinetics, and the over-expression of stemness genes (NANOG, SOX2, OCT4a), all confirmed that hAEC features and stemness potential are preserved in serum-free conditions. In particular, we proved to maintain a strong proliferation rate in serum-free conditions without affecting the gene expression profile of proliferation marker Ki67.

In the second phase of our study, we focused on the generation of $3 \mathrm{D}$ spheroids from hAECs cultured (a)

(b)
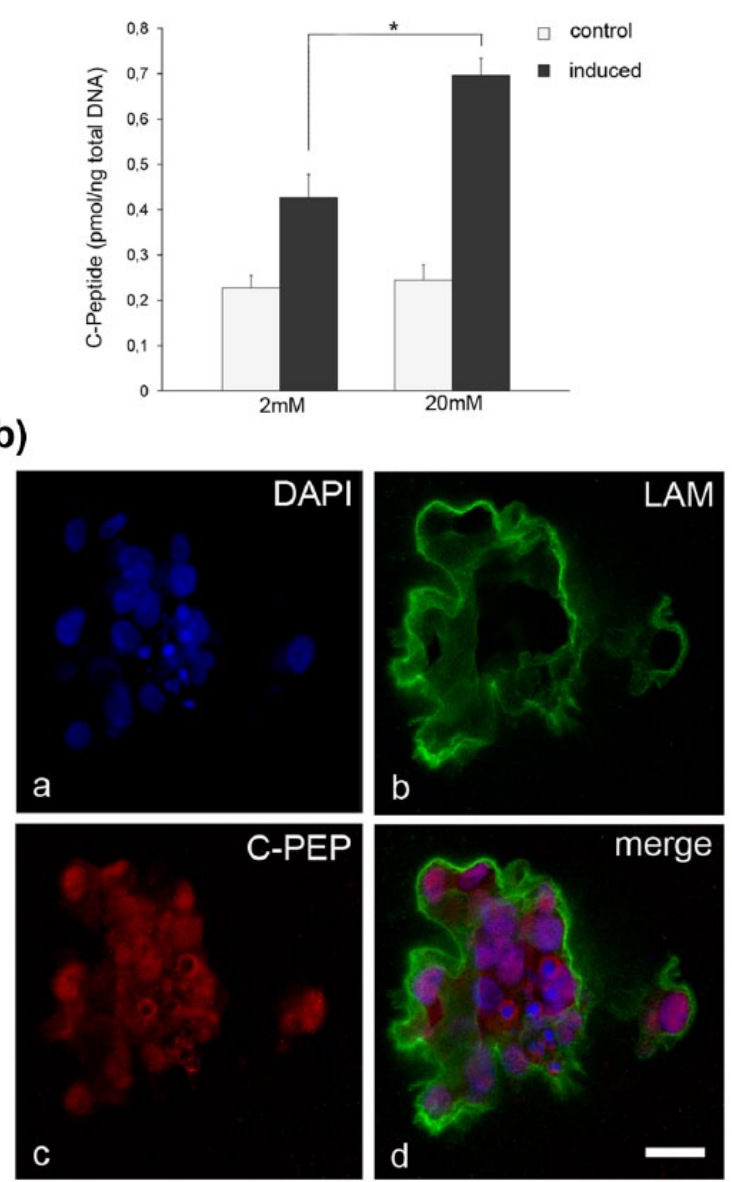

Figure 6. Glucose sensing and C-peptide release/ expression in vitro. (A) C-peptide release by hAEC spheroids in response to hypoglycemic (2 mM D-Glucose) and hyperglycemic (20 mM D-Glucose) conditions. C-peptide levels were measured by ELISA and normalized to total DNA content. Non-differentiated hAEC spheroids secreted a basal amount of $\mathrm{C}$-peptide in both conditions, while exposure to hyperglycemic conditions significantly increased C-peptide release in differentiated hAEC spheroids. Means \pm SD; $n=4$. $* P<0.02$. (b) Confocal immunofluorescence analysis of hAEC spheroids. Differentiated cells exposed to $20 \mathrm{mM}$ D-Glucose show a widespread C-peptide (c) expression. Laminin (b) staining highlights its enveloping function at the spheroids perimeter. Merge micrograph is shown (d). Nuclei are counterstained with DAPI (a). Scale bar $=20 \mu \mathrm{m}$.

on ECM. The vast majority of cell culture assays are still performed on synthetic substrates which force cells to adapt to a flat and rigid surface. Monolayer cultures compared to 3D cultures present several limitations concerning: viability, morphology, proliferation, response to stimuli, gene and protein expression, and differentiation efficiency. ${ }^{24-26}$ Hence, 
two-dimensional (2D) culture systems scarcely reproduce the in vivo environment and may, thus, have limited predictive value for clinical applications. A more physiological context is afforded by 3D culture methods which are more suitable for creating an adequate number of intercellular contacts and maintaining the in vivo phenotype and functionality. ${ }^{27,28}$ In the last decade, several reports described the generation of higher-order structures (embryoid bodies, glandular-like acini) from stem cells grown in matrices or other 3D supports. ${ }^{16,27,29}$ These studies have consistently increased our knowledge of several biological issues such as organ/tissue morphogenesis, cell growth, cancer development, and drug resistance. ${ }^{30}$ Therefore, despite the complex handling of 3D culture procedures, assays performed on a 3D system hold higher consistency than any $2 \mathrm{D}$ approach. ${ }^{19}$ In the present study we successfully show, for the first time, the generation of 3D spheroids obtained from placenta-derived primary epithelial stem cells. hAEC spheroids micrographs showed the typical round-shaped cell aggregates. Laminin expression revealed the encircling distribution of the basement membrane around spheroids, which suggests the correct acquisition of epithelial apico-basal polarity. The correct establishment and maintenance of cell polarity are crucial for normal cell physiology.

At this stage, we tested whether in vitro 3D culture was a valid approach to expand and efficiently differentiate hAECs into IPCs. Stepwise differentiation protocols applied to monolayer culture systems still remain the most adopted solutions to

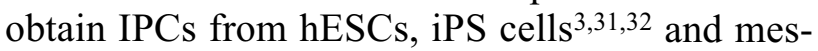
enchymal stem cells (MSCs). ${ }^{33}$ Recently, Takeuchi et al., ${ }^{21}$ showed that pancreatic-endocrine differentiation of hESCs cells cultured in monolayer, produced insulin-positive cells unable to secrete insulin in response to glucose stimulation, while 3D culture cell aggregates became functional IPCs. Although previous studies reported considerable evidence about successful insulin production from different stem cells, ${ }^{34}$ setting up an in vitro procedure to differentiate hAECs into IPCs has been a substantial task. We initially had performed direct differentiation with Nicotinamide as others reported, ${ }^{6,35}$ which only led to sporadic expression of insulin (data not shown) and no insulin secretion in response to glucose. Therefore, to emulate the in vivo pancreatic development pathway, we adapted, for our study on hAECs, the stepwise differentiation protocols from previous reports. ${ }^{11,31,32}$ SFM was used throughout the entire differentiation process and only three induction molecules were employed: Activin A, in the preinduction phase, to drive the endoderm formation; ${ }^{36}$ Nicotinamide (NAM), with Activin A in the second stage, to induce pancreatic-endocrine differentiation; ${ }^{37}$ and finally RA with NAM to promote IPC formation. ${ }^{38}$ Differentiated hAEC spheroids displayed insulin in the outer part of the spheroid and no consistent trace in the central core, where cells displayed an alpha cell phenotype (glucagon expression). Confocal micrographs revealed a defined cell membrane-associated localization of insulin. Here, as shown by TEM analysis, mature insulin-containing granules are gathered and eventually release their hormone content. C-peptide analysis undoubtedly confirmed the synthesis of insulin and revealed the glucose-dependent insulin secretion. The data showed the presence and production of insulin from a relatively limited number of hAECs $(60,000$ cells plated for each condition) and the fold increase of hormone produced by differentiated hAEC spheroids is comparable to analogous in vitro studies performed with adult stem cells. ${ }^{39}$

One of the most important characteristics required from candidate stem cells for regenerative medicine, is the ability to not elicit any immune response when transplanted. Culture procedures (passages, differentiation, 3D cultures) might compromise the immunomodulatory activity of cells that become no longer suitable for cell therapy approaches. Thus, besides the evaluations that led us to the derivation of insulin-producing cells, we simultaneously tested hAECs for their immunomodulatory properties in vitro. During serumfree validation we showed that FBS deprivation does not alter hAEC ability to inhibit PHA-activated PBMC proliferation in monolayer co-cultures. The same scenario was detected in hAEC spheroids: comparison between non-differentiated hAEC spheroids and differentiated hAEC spheroids (Figure 4) showed the same robust immunomodulation. Hence, hAEC spheroids maintained the immunomodulatory properties observed in monolayer cultures. Collectively, our results suggested that neither the cell transcriptome variations from $2 \mathrm{D}$ to $3 \mathrm{D}$ culture, nor the secretome modifications ${ }^{40}$ upon differentiation altered the immunogenicity of 
hAECs, which might, therefore, be suitable for transplantation.

In sum, our findings show the first attempt of hAEC pancreatic-endocrine differentiation, performed with the combination of serum-free culture condition, 3D multicellular spheroids creation, and stepwise differentiation protocol. The encouraging outcomes presented here usefully contribute to the implementation of similar procedures for in-depth in vitro and in vivo experiments, and highlight the role of hAECs as a possible cell therapy source for incurable diseases like diabetes.

\section{Acknowledgements}

We thank Cinzia Restani and Mariangela Ferrari for their valuable technical assistance.

\section{Author contributions}

$\mathrm{BO}$ and LI conceived, designed the study, and supervised the experiments. $\mathrm{BO}, \mathrm{RC}, \mathrm{DQ}$, and $\mathrm{FR}$ performed the experiments. BO, FA, RC, and LI interpreted data and performed statistical analysis. BO, FA, and RC wrote the manuscript. PP, LI, MD, DQ, and LB provided materials, reagents, and analysis tools. All authors have seen and approved the final version of the manuscript.

\section{Declaration of conflicting interests}

The author(s) declared no potential conflicts of interest with respect to the research, authorship, and/or publication of this article.

\section{Funding}

This research received no specific grant from any funding agency in the public, commercial, or not-for-profit sectors.

\section{References}

1. Roche E, Reig JA, Campos A, et al. (2005) Insulinsecreting cells derived from stem cells: Clinical perspectives, hypes and hopes. Transplant Immunology 15: e113-129.

2. Bonner Weir S and Weir GC (2005) New sources of pancreatic beta-cells. Nature Biotechnology 23: 857861.

3. Zhang D, Jiang W, Liu M, et al. (2009) Highly efficient differentiation of human ES cells and iPS cells into mature pancreatic insulin-producing cells. Cell Research 19: 429-438.

4. Lee K, Gan S and Calne R (2012) Stem cell therapy for diabetes. Indian Journal of Endocrinology and Metabolism 16: S227-229.

5. Parolini O, Alviano F, Bagnara GP, et al (2008) Concise review: Isolation and characterization of cells from human term placenta. Outcome of the first international Workshop on Placenta Derived Stem Cells. Stem Cells 26: 300-311.

6. Miki T, Lehmann T, Cai H, et al. (2005) Stem cell characteristics of amniotic epithelial cells. Stem Cells 23: $1549-1559$.

7. Hori J, Wang M, Kamiya K, et al. (2006) Immunological characteristics of amniotic epithelium. Cornea 25 (Suppl. 1): S53-58.

8. Bilic G, Zeisberger SM, Mallik AS, et al. (2008) Comparative characterization of cultured human term amnion epithelial and mesenchymal stromal cells for application in cell therapy. Cell Transplantation 17: 955-968.

9. Sakuragawa N, Misawa H, Ohsugi K, et al. (1997) Evidence for active acetylcholine metabolism in human amniotic epithelial cells: Applicable to intracerebral allografting for neurologic disease. Neuroscience Letters 232: 53-56.

10. Marongiu F, Gramignoli R, Dorko K, et al. (2011) Hepatic differentiation of amniotic epithelial cells. Hepatology 53: 1719-1729.

11. Bhandari DR, Seo KW, Sun B, et al. (2011) The simplest method for in vitro $\beta$-cell production from human adult stem cells. Differentiation 82: 144-152.

12. Banas RA, Trumpower C, Bentlejewski C, et al. (2008) Immunogenicity and immunomodulatory effects of amnion-derived multipotent progenitor cells. Human Immunology 69: 321-328.

13. Wolbank S, Peterbauer A, Fahrner M, et al. (2007) Dose-dependent immunomodulatory effect of human stem cells from amniotic membrane: A comparison with human mesenchymal stem cells from adipose tissue. Tissue Engineering 13: 1173-1183.

14. Sensebé L, Bourin P and Tarte K (2011) Good manufacturing practices production of mesenchymal stem/ stromal cells. Human Gene Therapy 22: 19-26.

15. van der Valk J, Brunner D, De Smet K, et al. (2010) Optimization of chemically defined cell culture media-replacing fetal bovine serum in mammalian in vitro methods. Toxicology In Vitro 24: 1053-1063.

16. Debnath J, Muthuswamy SK and Brugge JS (2003) Morphogenesis and oncogenesis of MCF-10A mammary epithelial acini grown in three-dimensional basement membrane cultures. Methods 30: 256-268.

17. Fennema E, Rivron N, Rouwkema J, et al. (2013) Spheroid culture as a tool for creating 3D complex tissues. Trends in Biotechnology 31: 108-115.

18. Goh SK, Olsen P and Banerjee I (2013) Extracellular matrix aggregates from differentiating embryoid bodies as a scaffold to support ESC proliferation and differentiation. PLoS One 8: e61856.

19. Benton G, George J, Kleinman HK, et al. (2009) Advancing science and technology via $3 \mathrm{D}$ culture 
on basement membrane matrix. Journal of Cell Physiology 221: 18-25.

20. Parekkadan B, Berdichevsky Y, Irimia D, et al. (2008) Cell-cell interaction modulates neuroectodermal specification of embryonic stem cells. Neuroscience Letters 438: 190-195.

21. Takeuchi H, Nakatsuji N and Suemori H (2014) Endodermal differentiation of human pluripotent stem cells to insulin-producing cells in 3D culture. Scientific Reports 4: 4488.

22. Ilancheran S, Michalska A, Peh G, et al. (2007) Stem cells derived from human fetal membranes display multi-lineage differentiation potential. Biology of Reproduction 77: 577-588.

23. Cao LZ, Tang DQ, Horb ME, et al. (2004) High glucose is necessary for complete maturation of pdx-1-vp16-expressing hepatic cells into functional insulin-producing cells. Diabetes 53: 3168.

24. Li Y, Ma T, Kniss DA, et al. (2001) Human cord cell hematopoiesis in three-dimensional nonwoven fibrous matrices: In vitro simulation of the marrow microenvironment. Journal of Hematotherapy and Stem Cell Research 10: 355-368.

25. Bokhari M, Carnachan RJ, Cameron NR, et al. (2007) Culture of HepG2 liver cells on three dimensional polystyrene scaffolds enhances cell structure and function during toxicological challenge. Journal of Anatomy 211: 567-576.

26. Tian XF, Heng BC, Ge Z, et al. (2008) Comparison of osteogenesis of human embryonic stem cells within 2D and 3D culture systems. Scandinavian Journal of Clinical \& Laboratory Investigation 68: 58-67.

27. Meng X, Leslie P, Zhang Y, et al. (2014) Stem cells in a three-dimensional scaffold environment. Springerplus $11 ; 80$.

28. Benton G, Crooke E and George J (2009) Laminin-1 induces E-cadherin expression in 3-dimensional cultured breast cancer cells by inhibiting DNA methyltransferase 1 and reversing promoter methylation status. FASEB Journal 23: 3884-3895.

29. Eritja N, Llobet D, Domingo M, et al. (2010) A novel three-dimensional culture system of polarized epithelial cells to study endometrial carcinogenesis. American Journal of Pathology 176: 2722-2731.
30. Bates RC, Edwards NS and Yates JD (2000) Spheroids and cell survival. Critical Reviews in Oncology/ Hematology 36: 61-74.

31. D'Amour KA, Bang AG, Eliazer S, et al. (2006) Production of pancreatic hormone-expressing endocrine cells from human embryonic stem cells. Nature Biotechnology 24: 1392-1401.

32. Kroon E, Martinson LA, Kadoya K, et al. (2008) Pancreatic endoderm derived from human embryonic stem cells generates glucose-responsive insulin-secreting cells in vivo. Nature Biotechnology 26: 443-452.

33. Prabakar KR, Domínguez-Bendala J, Molano RD, et al. (2012) Generation of glucose-responsive, insulin-producing cells from human umbilical cord bloodderived mesenchymal stem cells. Cell Transplantation 21: 1321-1339.

34. Zulewski H (2006) Stem cells with potential to generate insulin producing cells in man. Swiss Medical Weekly 136: 647-654.

35. Vaca P, Berná G, Araujo R, et al. (2008) Nicotinamide induces differentiation of embryonic stem cells into insulin-secreting cells. Experimental Cell Research 314: 969-974.

36. D'Amour KA, Agulnick AD, Eliazer S, et al. (2005) Efficient differentiation of human embryonic stem cells to definitive endoderm. Nature Biotechnology 23: $1534-1541$.

37. Otonkoski T, Beattie GM, Mally MI, et al. (1993) Nicotinamide is a potent inducer of endocrine differentiation in cultured human fetal pancreatic cells. Journal of Clinical Investigation 92: 1459-1466.

38. Oström M, Loffler KA, Edfalk S, et al. (2008) Retinoic acid promotes the generation of pancreatic endocrine progenitor cells and their further differentiation into beta-cells. PLoS One 3: e2841.

39. Chandra V, Swetha G, Muthyala S, et al. (2011) Isletlike cell aggregates generated from human adipose tissue derived stem cells ameliorate experimental diabetes in mice. PLoS One 6: e20615.

40. Tee JY, Vaghjiani V, Liu YH, et al. (2013) Immunogenicity and immunomodulatory properties of hepatocyte-like cells derived from human amniotic epithelial cells. Current Stem Cell Research \& Therapy 8: 91-99. 